\title{
Thalassiosirales (Diatomeae) do rio Guaraguaçu, Bacia Litorânea, PR, Brasil
}

\author{
Priscila Izabel Tremarin ${ }^{1,2}$, Thelma Alvim Veiga Ludwig ${ }^{1}$ e Hermes Moreira Filho ${ }^{1}$
}

Recebido em 29/08/2007. Aceito em 3/04/2008

\begin{abstract}
RESUMO - (Thalassiosirales (Diatomeae) do rio Guaraguaçu, Bacia Litorânea, PR, Brasil). Realizou-se o inventário florístico das diatomáceas pertencentes à ordem Thalassiosirales do rio Guaraguaçu. Amostras planctônicas e perifíticas foram coletadas em seis estações ao longo do leito do rio, considerando um gradiente de salinidade sob influência da maré, rio adentro. A análise qualitativa do material permitiu a identificação de 28 espécies, sendo 19 pertencentes à família Thalassiosiraceae. Do total de táxons determinados registraram-se 85,7\% marinhas/estuarinas e 14,3\% dulcícolas. Cyclotella atomus Hustedt, C. choctawhatcheeana Prasad, Thalassiocyclus lucens (Hustedt) Hakansson \& Mahood, Thalassiosira minima Gaarder, T. prochkinae Makarova, T. pseudonana Hasle \& Heimdal, T. tealata Takano, T. tenera Proschkina-Lavrenko e Thalassiosira sp. foram os nove novos registros para o Estado do Paraná. Chaves de identificação, descrições, medidas, ilustrações em microscopia óptica e eletrônica de varredura complementam o estudo.
\end{abstract}

Palavras-chave: ambiente lótico, Coscinodiscophyceae, diatomáceas, estuário, taxonomia

\begin{abstract}
Thalassiosirales (Diatomeae) of the Guaraguaçu river, littoral basin, Paraná State, Brazil). The floristic inventory of Thalassiosirales from Guaraguaçu river was based on planktonic and periphytic samples collected at six sampling stations throughout the river, considering a salinity gradient. A total of 28 species were identified with 19 included in Thalassiosiraceae family. Among the diatoms recorded, $85,7 \%$ were marine/estuarine and 14,3\% were continental species. Cyclotella atomus Hustedt, C. choctawhatcheeana Prasad, Thalassiocyclus lucens (Hustedt) Hakansson \& Mahood, Thalassiosira minima Gaarder, T. prochkinae Makarova, T. pseudonana Hasle \& Heimdal, T. tealata Takano, T. tenera Proschkina-Lavrenko and Thalassiosira sp. were the nine new records for the State of the Paraná. Identification keys, taxonomic descriptions, measurements, MO and MEV illustrations complement the study.
\end{abstract}

Key words: lotic system, Coscinodiscophyceae, diatoms, estuary, taxonomy

\section{Introdução}

O estudo da flora diatomológica de ambientes lóticos continentais do Estado do Paraná iniciou-se com os trabalhos de Moreira Filho et al. (1973) e Contin (1990) no rio Iguaçu. Lozovei \& Shirata (1990) encontraram 152 espécies de diatomáceas em amostras do rio Passaúna. Posteriormente, foram realizados os trabalhos de Ludwig \& Flôres $(1995 ; 1997)$ em rios da área de abrangência da usina hidrelétrica de Segredo e os de Brassac et al. (1999) e Brassac \& Ludwig (2005; 2006) em rios a serem represados para a construção da usina hidrelétrica de Salto Caxias. A diversidade do fitoplâncton do rio Tibagi, próximo ao município de Londrina, foi analisada por Bittencourt-Oliveira (2002) e Leandrini et al. (2002) estudaram as espécies perifíticas de Navicula em dois sistemas lóticos do município de Maringá. Poucos trabalhos foram realizados em ambientes lóticos no litoral paranaense, destacando-se o de Landucci \& Ludwig (2005) que registraram 20 espécies das classes Coscinodiscophyceae e Fragilariophyceae em nove rios da região.

Estudos sobre a diatomoflora de ecossistemas reófilos estuarinos do Paraná são inexistentes. O rio Guaraguaçu é um ecossistema litorâneo paranaense importante pela sua dimensão e volume d'água. À medida que o corpo de água se aproxima da foz do rio, a água doce mistura-se gradualmente com a água salgada do oceano adjacente pela ação do ciclo de marés, caracterizando um ambiente estuarino.

O trabalho teve por objetivo realizar o estudo taxonômico das diatomáceas pertencentes à ordem Thalassiosirales em amostras coletadas no rio Guaraguaçu, registrando-se a variabilidade morfológica e métrica das

\footnotetext{
1 Universidade Federal do Paraná, Departamento de Botânica, Laboratório de Ficologia, C. Postal 19031, Centro Politécnico, 81531-990 Curitiba, PR, Brasil

2 Autor para correspondência: ptremarin@gmail.com
} 
diferentes espécies, contribuindo-se para o inventário da diatomoflora de ambientes lóticos paranaenses.

\section{Material e métodos}

O rio Guaraguaçu está localizado na bacia hidrográfica litorânea paranaense, nos municípios de Pontal do Paraná, Paranaguá e Matinhos, Estado do Paraná, sul do Brasil. Vinte e nove amostras contendo material planctônico e perifítico foram coletadas em seis

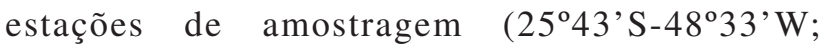
$25^{\circ} 40^{\prime}$ 'S-48 $30^{\prime} \mathrm{W}$; $25^{\circ} 39^{\prime} \mathrm{S}-48^{\circ} 30^{\prime} \mathrm{W}$; $25^{\circ} 37^{\prime}$ 'S-48 $30^{\circ}$ 'W; $25^{\circ} 36^{\prime} \mathrm{S}-48^{\circ} 29^{\prime} \mathrm{W}, 25^{\circ} 35^{\prime} \mathrm{S}-48^{\circ} 29^{\prime} \mathrm{W}$ ). A coleta foi realizada nos meses de abril e outubro de 2003 (Tab.1), em período de maré baixa e alta. As amostras planctônicas foram coletadas com auxílio de rede de plâncton com abertura de malha de $25 \mu \mathrm{m}$ e as de perifíton por meio de raspagem de partes de macrófitas aquáticas submersas coletadas, todas encontram-se registradas no Herbário da Universidade Federal do Paraná (UPCB). O material foi oxidado segundo a técnica de Simonsen (1974) modificada por Moreira Filho \& Valente-Moreira (1981). Para a análise do material em Microscopia Óptica (MO) foram confeccionadas lâminas permanentes com resina Naphrax ${ }^{\circledR}$ (I.R.: 1.74). Para a observação em Microscopia Eletrônica de Varredura (MEV), parte do material oxidado foi depositada sobre suportes de alumínio, seca em chapa de aquecimento e metalizada em aparelho Balser Sputtering/SDC300. Ilustrações em MO foram realizadas em fotomicroscópio Olympus BX40 com filme Imagelink da Kodak e papel Kodabromide F3 e em MEV em microscópio Jeol-JSM 6360.

A classificação baseou-se em Cavalier-Smith (1998) - subdivisão Diatomeae - e Round et al. (1990) categorias taxonômicas inferiores. A terminologia empregada nas descrições dos táxons seguiu Barber \& Haworth (1981) e Round et al. (1990).

\section{Resultados e discussão}

A análise qualitativa de 29 amostras provenientes do rio Guaraguaçu permitiu a identificação de 28 espécies de diatomáceas pertencentes às famílias Thalassiosiraceae (19), Stephanodiscaceae (7), Skeletonemataceae (1) e Lauderiaceae (1). Cyclotella atomus Hustedt, C. choctawhatcheeana Prasad, Thalassiocyclus lucens

Tabela 1. Informações sobre as coletas realizadas no rio Guaraguaçu, PR, Brasil.

\begin{tabular}{|c|c|c|c|c|c|c|}
\hline UPCB & Estação & Município & Salinidade & Habitat & Data & Coletores \\
\hline 47493 & 2 & Pontal do Paraná & 0 & Plâncton & $16 / \mathrm{IV} / 2003$ & P. Bigunas, T. Ludwig \& A. Campos \\
\hline 47494 & 2 & Pontal do Paraná & 0 & Perifíton & $16 / \mathrm{IV} / 2003$ & P. Bigunas, T. Ludwig \& A. Campos \\
\hline 47495 & 3 & Pontal do Paraná & 0 & Plâncton & 16/IV/2003 & P. Bigunas, T. Ludwig \& A. Campos \\
\hline 47496 & 3 & Pontal do Paraná & 0 & Perifíton & $16 / \mathrm{IV} / 2003$ & P. Bigunas, T. Ludwig \& A. Campos \\
\hline 47497 & 4 & Pontal do Paraná & 0 & Plâncton & 16/IV/2003 & P. Bigunas, T. Ludwig \& A. Campos \\
\hline 47498 & 4 & Pontal do Paraná & 0 & Perifíton & 16/IV/2003 & P. Bigunas, T. Ludwig \& A. Campos \\
\hline 47499 & 4 & Pontal do Paraná & 2 & Plâncton & $16 / \mathrm{IV} / 2003$ & P. Bigunas, T. Ludwig \& A. Campos \\
\hline 47500 & 4 & Pontal do Paraná & 0 & Perifíton & $16 / \mathrm{IV} / 2003$ & P. Bigunas, T. Ludwig \& A. Campos \\
\hline 47501 & 5 & Pontal do Paraná & 10 & Plâncton & 16/IV/2003 & P. Bigunas, T. Ludwig \& A. Campos \\
\hline 47502 & 5 & Pontal do Paraná & 4 & Plâncton & 16/IV/2003 & P. Bigunas, T. Ludwig \& A. Campos \\
\hline 47503 & 5 & Pontal do Paraná & 4 & Perifíton & 16/IV/2003 & P. Bigunas, T. Ludwig \& A. Campos \\
\hline 47506 & 6 & Pontal do Paraná & 13 & Perifíton & 16/IV/2003 & P. Bigunas, T. Ludwig \& A. Campos \\
\hline 47509 & 1 & Matinhos & 0 & Plâncton & $24 / X / 2003$ & P. Bigunas, T. Ludwig \& J. Silva \\
\hline 47510 & 1 & Matinhos & 0 & Perifíton & $24 / \mathrm{X} / 2003$ & P. Bigunas, T. Ludwig \& J. Silva \\
\hline 47511 & 1 & Matinhos & 0 & Perifíton & $24 / \mathrm{X} / 2003$ & P. Bigunas, T. Ludwig \& J. Silva \\
\hline 47512 & 2 & Pontal do Paraná & 0 & Plâncton & $24 / X / 2003$ & P. Bigunas, T. Ludwig \& J. Silva \\
\hline 47513 & 2 & Pontal do Paraná & 0 & Perifíton & $24 / \mathrm{X} / 2003$ & P. Bigunas, T. Ludwig \& J. Silva \\
\hline 47514 & 3 & Pontal do Paraná & 0 & Plâncton & 24/X/2003 & P. Bigunas, T. Ludwig \& J. Silva \\
\hline 47515 & 3 & Pontal do Paraná & 0 & Perifíton & $24 / X / 2003$ & P. Bigunas, T. Ludwig \& J. Silva \\
\hline 47516 & 3 & Pontal do Paraná & 0 & Perifíton & $24 / X / 2003$ & P. Bigunas, T. Ludwig \& J. Silva \\
\hline 47517 & 4 & Pontal do Paraná & 0 & Perifíton & $24 / X / 2003$ & P. Bigunas, T. Ludwig \& J. Silva \\
\hline 47518 & 4 & Pontal do Paraná & 0 & Perifíton & $24 / \mathrm{X} / 2003$ & P. Bigunas, T. Ludwig \& J. Silva \\
\hline 47519 & 5 & Pontal do Paraná & 0 & Plâncton & $24 / \mathrm{X} / 2003$ & P. Bigunas, T. Ludwig \& J. Silva \\
\hline 47520 & 5 & Pontal do Paraná & 0 & Perifíton & $24 / \mathrm{X} / 2003$ & P. Bigunas, T. Ludwig \& J. Silva \\
\hline 47521 & 6 & Pontal do Paraná & 0 & Plâncton & $24 / X / 2003$ & P. Bigunas, T. Ludwig \& J. Silva \\
\hline 47523 & 2 & Pontal do Paraná & 1 & Plâncton & $24 / X / 2003$ & P. Bigunas, T. Ludwig \& J. Silva \\
\hline 47524 & 3 & Pontal do Paraná & 1 & Plâncton & $24 / \mathrm{X} / 2003$ & P. Bigunas, T. Ludwig \& J. Silva \\
\hline 47525 & 4 & Pontal do Paraná & 1 & Plâncton & $24 / \mathrm{X} / 2003$ & P. Bigunas, T. Ludwig \& J. Silva \\
\hline 47526 & 5 & Pontal do Paraná & 14 & Plâncton & $24 / \mathrm{X} / 2003$ & P. Bigunas, T. Ludwig \& J. Silva \\
\hline
\end{tabular}


(Hustedt) Hakansson \& Mahood, Thalassiosira minima Gaarder, T. prochkinae Makarova, T. pseudonana Hasle \& Heimdal, T. tealata Takano, T. tenera ProschkinaLavrenko e Thalassiosira sp. resultaram em registros pioneiros para o Estado.

Os gêneros Thalassiocyclus, Cymatotheca, Cymatodiscus e Tryblioptychus não estão citados no sistema de classificação proposto por Round et al. (1990). Entretanto, por apresentarem aréolas loculadas ocluídas por cribra, anel marginal de fultopórtulas e uma rimopórtula, características morfológicas importantes de Thalassiosiraceae, foram incluídos nesta família, como sugerem Prasad et al. (2002) e Håkansson \& Mahood (1993).

A maioria das espécies $(85,7 \%)$ encontradas no estudo apresenta hábito salobro a marinho indicando que o rio Guaraguaçu sofre influência da baía adjacente. Espécies marinhas e salobras presentes na baía de Paranaguá provavelmente foram carreadas para o interior do rio pelo movimento de maré, alterando parte da diatomoflórula do Guaraguaçu.

\section{COSCINODISCOPHYCEAE \\ THALASSIOSIRALES THALASSIOSIRACEAE}

\section{Cymatodiscus Hendey}

Cymatodiscus planetophorus (Meister) Hendey, J. Roy. Microsc. Soc. 77(1): 42. 1958.

Coscinodiscus planetophorus Meister, Kiesel. Asien, p. 34, fig., 86-87, 1932.

Fig. 1-2

Valvas elípticas, assimétricas; superfície valvar plana; aréolas esparsas próximas à região central e dispostas em fileiras radiadas próximo às margens; presença de uma fileira de fultopórtulas marginais interrompida por uma rimopórtula de difícil visualização. Compr.: 17,4-26,1 $\mu \mathrm{m}$; larg.: 12,6-17,4 $\mu \mathrm{m}$; 11-16 aréolas em $10 \mu \mathrm{m} ; 2$ fultopórtulas em $10 \mu \mathrm{m}$.

Material examinado: Brasil. Paraná: Pontal do Paraná, rio Guaraguaçu, 16/IV/2003, P. Bigunas, T. Ludwig \& A. Campos (UPCB 47499, 47501, 47503, 47506); 24/X/2003, P. Bigunas, T. Ludwig \& J. Silva (UPCB 47515, 47525, 47520, 47526).

\section{Cymatotheca Hendey}

Cymatotheca weissflogii (Grunow) Hendey, J. Roy. Microsc. Soc. 77(3): 41, pl. 5, fig. 9, 1958.

Euodia weissflogii Grunow in Van Heurck, Syn. Diat.

Belg., pl. 126, fig. 13, 1883.

Fig. 3-4, 60

Valvas elípticas; superfície valvar ondulada longitudinalmente; aréolas dispostas em fileiras radiadas, mais grosseiras em um dos lados da valva; presença de uma fileira de fultopórtulas marginais interrompida por uma rimopórtula de difícil visualização. Compr.: 8,7-25,3 $\mu \mathrm{m}$; larg.: 8,7-20,5 $\mu \mathrm{m} ; 10-12$ aréolas em $10 \mu \mathrm{m} ; 2$ fultopórtulas em $10 \mu \mathrm{m}$.

Material examinado: Brasil. Paraná: Pontal do Paraná, rio Guaraguaçu, 16/IV/2003, P. Bigunas, T. Ludwig \& A. Campos (UPCB 47493, 47494, 47495, 47496, 47497, 47498, 47499, 47501, 47502, 47503); 24/X/2003, P. Bigunas, T. Ludwig \& J. Silva (UPCB 47509, 47510, 47511, 47512, 47513, 47523, 47514, 47515, 47524, 47516, 47517, 47518, 47525, 47519, 47520, 47526).

Minidiscus Hasle

Chave para as espécies de Minidiscus

1. Superfície valvar ondulada na porção central da valva; rimopórtula entre duas fultopórtulas 1. M. chilensis

1. Superfície valvar levemente convexa; rimopórtula rodeada por fultopórtulas

2. M. comicus

1. Minidiscus chilensis Rivera, in Rivera \& Koch, Proceedings of the $7^{\text {th }}$ International Diatom Symposium, p. 281, 1982.

Fig. 5-8, 61

Valvas circulares; superfície valvar ondulada centralmente; aréolas inconspícuas; presença de três fultopórtulas, duas na elevação e uma próxima à depressão valvar; uma rimopórtula entre duas fultopórtulas. Diâm.: 6-8,7 $\mu \mathrm{m}$.

Atualmente são conhecidas sete espécies para o gênero: $M$. comicus, $M$. chilensis, $M$. trioculatus (Taylor) Hasle, M. decoratus Chrétiennot-Dinet \& Quiroga, M. spinulosus Gao, Chang \& Chin, M. occelatus Gao, Chang \& Chin e M. subtilis Gao, Chang \& Chin (Aké-Castillo et al. 2001). Em águas brasileiras, apenas M. comicus, M. chilensis foram registradas (Fernandes et al. 1999; Brandini et al. 2001; Procopiak et al. 2006).

Quiroga \& Chrétiennot-Dinet (2004) comentaram que a observação das valvas de Minidiscus em microscopia eletrônica geralmente é necessária para a correta determinação das espécies uma vez que estas apresentam tamanho diminuto.

Material examinado: Brasil. Paraná: Pontal do Paraná, rio Guaraguaçu, 16/IV/2003, P. Bigunas, T. Ludwig \& A. Campos (UPCB 47502, 47506); 24/X/2003, P. Bigunas, T. Ludwig \& J. Silva (UPCB 47520, 47521).

2. Minidiscus comicus Takano, Bull. Tokai Reg. Fish. Res. Lab., (105): 31, fig. 1A, 2-13, 1981.

Fig. 9 
Valvas circulares; superfície valvar levemente convexa; margem valvar hialina ausente ou estreita; aréolas inconspícuas; presença de três fultopórtulas ao redor de uma rimopórtula situada próximo ao centro valvar. Diâm.: 4-6,2 $\mu \mathrm{m}$.

Além das diferenças constadas em MO (disposição das estruturas perfuradas e presença ou não de ondulação da superfície valvar), $M$. comicus e $M$. chilensis também apresentam características ultra-estruturais distintivas na superfície valvar. $M$. comicus possui aréolas em toda superfície valvar e três poros satélites em cada fultopórtula, enquanto que $M$. chilensis apresenta aréolas restritas à margem valvar, apenas dois poros satélites por fultopórtula e um poro central ou subcentral ocluído por sílica (Aké-Castillo et al. 2001).

Material examinado: Brasil. Paraná: Pontal do Paraná, rio Guaraguaçu, 16/IV/2003, P. Bigunas, T. Ludwig \& A. Campos (UPCB 47498, 47500, 47501, 47502, 47503, 47506); 24/X/2003, P. Bigunas, T. Ludwig \& J. Silva (UPCB 47509, 47510, 47516, 47518, 47525, 47519, 47520, 47526, 47521).

Thalassiocyclus Håkansson \& Mahood
Thalassiocyclus lucens (Hustedt) Hakansson \& Mahood, Nova Hedwigia 106: 198, 1993.

Stephanodiscus lucens Hustedt, Abh. Naturw. Ver. Bremen 31(3): 583-584, fig. 4, 1939.

Fig. 10

Valvas circulares; superfície valvar ondulada na porção central; aréolas inconspícuas; presença de uma fultopórtula próxima à região central da valva; anel marginal de fultopórtulas interrompido por uma rimopórtula delicada. Diâm.: 4,7-5,8 $\mu$ m; 8 fultopórtulas em $10 \mu \mathrm{m}$.

Valvas onduladas transversalmente e radialmente, com uma fultopórtula delicada ao término de interfascículos ramificados são algumas das características que distinguem Thalassiocyclus dos demais gêneros de Thalassiosiraceae. A única espécie do gênero, T. lucens, ocorre em águas salobras com pH próximo ao neutro (Håkansson \& Mohood 1993).

Material examinado: Brasil. Paraná: Pontal do Paraná, rio Guaraguaçu, 24/X/2003, P. Bigunas, T. Ludwig \& J. Silva (UPCB 47512, 47514, 47519).

\section{Thalassiosira Cleve}

Chave para as espécies de Thalassiosira

1. Rimopórtula central ou subcentral

2. Aréolas grosseiras, cerca de 5-8 em $10 \mu \mathrm{m}$ 7. T. oestrupii

2. Aréolas delicadas, às vezes inconspícuas, cerca de 16-20 em $10 \mu \mathrm{m}$

8. T. proschkinae

1. Rimopórtula marginal

3. Fileira dupla de fultopórtulas marginais

2. T. eccentrica

3. Fileira única de fultopórtulas marginais

4. Presença de fultopórtula central ou subcentral

5. Aréolas conspícuas

6. Aréolas dispostas em fileiras diagonais

7. Valvas maiores que $19 \mu \mathrm{m}$; aréola central de igual tamanho que as demais ....5. T. nanolineata

7. Valvas menores que $8 \mu \mathrm{m}$; aréola central levemente maior que as demais 12. T. tenera

6. Aréolas dispostas em fileiras radiais ou tangenciais

8. Presença de costelas marginais delicadas

10. T. punctigera

8. Ausência de costelas marginais

9. Valvas com 5-10 fultopórtulas marginais em $10 \mu \mathrm{m}$ 1. T. decipiens

9. Valvas com 3-4 fultopórtulas marginais em $10 \mu \mathrm{m}$

5. Aréolas inconspícuas

10. Uma fultopórtula próxima ao centro valvar

11. T. tealata

10. Duas fultopórtulas próximas ao centro valvar

4. T. minima

4. Ausência de fultopórtula central ou subcentral

11. Duas rimopórtulas marginais

13. Thalassiosira sp.

11. Uma rimopórtula marginal

12. Valvas maiores que $20 \mu \mathrm{m}$; aréolas conspícuas

3. T. leptopus

12. Valvas menores que $5 \mu \mathrm{m}$; aréolas inconspícuas 9. T. pseudonana 
1. Thalassiosira decipiens (Grunow) E. Joergensen in O. Nordgaard, Hydr. Biol. Invest. Norw. Fior., p. 96, pl. 6, fig. 3, 1905.

Coscinodiscus eccentricus var. decipiens Grunow, Alg. Diat. Kasp. Meer., p. 125, pl. 4, fig. 18, 1878.

Fig. 11

Valvas circulares; superfície valvar côncava; aréolas hexagonais dispostas em fileiras tangenciais; presença de uma fultopórtula central e fileira única de fultopórtulas marginais; uma rimopórtula marginal. Diâm.: 8,7-19,8 $\mu \mathrm{m} ; 8$-12 aréolas em $10 \mu \mathrm{m} ; 5-10$ fultopórtulas marginais em $10 \mu \mathrm{m}$.

Material examinado: Brasil. Paraná: Pontal do Paraná, rio Guaraguaçu, 16/IV/2003, P. Bigunas, T. Ludwig \& A. Campos (UPCB 47494, 47498, 47502, 47506); 24/X/2003, P. Bigunas, T. Ludwig \& J. Silva (UPCB 47509, 47512, 47513, 47514, 47515, 47516, 47517, 47518, 47519, 47520, 47526, 47521).

2. Thalassiosira eccentrica (Ehrenberg) Cleve, Bull. Couns. Explor. Mer., p. 216, 1903 (1904).

Coscinodiscus excentricus Ehrenberg, Abh. Akad. Wiss.

Ber., p. 146, 1839, p. 371, pl. 3, fig., 5, 1841 (1843).

Fig. 12

Valvas circulares; superfície valvar plana; aréolas hexagonais dispostas em fileiras tangenciais; presença de fultopórtulas distribuídas pela superfície valvar e de fileira dupla de fultopórtulas marginais; uma rimopórtula marginal pouco evidente. Diâm.: 39,5-51,4 $\mu$ m; 5 aréolas em $10 \mu \mathrm{m} ; 8$ fultopórtulas marginais em $10 \mu \mathrm{m}$.

Material examinado: Brasil. Paraná: Pontal do Paraná, rio Guaraguaçu, 16/IV/2003, P. Bigunas, T. Ludwig \& A. Campos (UPCB 47493, 47495, 47497, 47502, 47506); 24/X/2003, P. Bigunas, T. Ludwig \& J. Silva (UPCB 47513, 47514, 47524, 47516, 47525, 47520, 47526).

3. Thalassiosira leptopus (Grunow) Hasle \& Fryxell, Nova Hedwigia, 54: 20, pl. 1, fig. 1-4, pl. 2, fig. 5-9, pl. 3, fig. 10-12, pl. 4, fig. 13-14, pl. 18, fig. 94-96, 1976.

Coscinodiscus lineatus Ehrenberg var. leptopus Grunow in Van Heurck, Syn. Diat. Belg., pl. 131, fig. 5-6, 1883.

Fig. 13-15

Valvas circulares; superfície valvar plana; aréolas hexagonais dispostas em fileiras diagonais; fileira de fultopórtulas marginais de difícil visualização; uma rimopórtula marginal. Diâm.: 20,5-29,2 $\mu$ m; 10-14 aréolas em $10 \mu \mathrm{m}$; 4-6 fultopórtulas marginais em $10 \mu \mathrm{m}$.

Material examinado: Brasil. Paraná: Pontal do Paraná, rio Guaraguaçu, 16/IV/2003, P. Bigunas, T. Ludwig \& A. Campos (UPCB 47502, 47506); 24/X/2003, P. Bigunas, T. Ludwig \& J. Silva (UPCB
47510, 47512, 47513, 47523, 47514, 47515, 47524, 47516, 47517, 47518, 47525, 47519, 47520, 47521).

4. Thalassiosira minima Gaarder, Rep. Sci. Res. Nor. Atl. Exp., 2(2): 31, fig. 18, 1951.

Fig. 16-17

Valvas circulares; superfície valvar plana; aréolas inconspícuas; duas fultopórtulas centrais e fileira única de fultopórtulas marginais; uma rimopórtula marginal. Diâm.: 5,9 $\mu \mathrm{m}$, aréolas inconspícuas, 4 fultopórtulas em $10 \mu \mathrm{m}$.

Material examinado: Brasil. Paraná: Pontal do Paraná, rio Guaraguaçu, 24/X/2003, P. Bigunas, T. Ludwig \& J. Silva (UPCB 47526).

5. Thalassiosira nanolineata (Mann) Fryxell \& Hasle, Nova Hedwigia (54): 32, fig. 74-80, 1977.

Coscinodiscus nanolineatus Mann, Bull. U. S. Nat. Mus., 100(6/1): 68, pl. 14, fig. 4, 1925.

Fig. 18-19

Valvas circulares; superfície valvar convexa; aréolas hexagonais dispostas em fileiras diagonais; uma a três fultopórtulas no centro da valva; fileira de fultopórtulas marginais interrompida por uma rimopórtula. Diâm.: 19-31,6 $\mu \mathrm{m} ; 5-6$ aréolas em $10 \mu \mathrm{m}$; 5-8 fultopórtulas marginais em $10 \mu \mathrm{m}$.

Material examinado: Brasil. Paraná: Pontal do Paraná, rio Guaraguaçu, 16/IV/2003, P. Bigunas, T. Ludwig \& A. Campos (UPCB 47493, 47494, 47495, 47496, 47497, 47498, 47499, 47502, 47506); 24/X/2003, P. Bigunas, T. Ludwig \& J. Silva (UPCB 47509, 47510, 47511, 47512, 47513, 47523, 47514, 47515, 47524, 47516, 47517, 47518, 47525, 47520, 47526).

6. Thalassiosira nordenskioeldii Cleve, Sven. Vet. Akad.

Handl. 1(13): 7, pl. 1, fig. 1, 1873.

Fig. 20-21

Valvas circulares; superfície valvar convexa; aréolas hexagonais arranjadas em fileiras tangenciais; uma fultopórtula central pouco evidente e fileira única de fultopórtulas marginais alongadas radialmente; rimopórtula marginal proeminente. Diâm.: 10,3-15 $\mu \mathrm{m} ; 12$ aréolas em $10 \mu \mathrm{m}$; 3-4 fultopórtulas marginais em $10 \mu \mathrm{m}$.

Material examinado: Brasil. Paraná: Pontal do Paraná, rio Guaraguaçu, 16/IV/2003, P. Bigunas, T. Ludwig \& A. Campos (UPCB 47494, 47495, 47496, 47497, 47498, 47499, 47503); 24/X/2003, P. Bigunas, T. Ludwig \& J. Silva (UPCB 47509, 47510, 47511, 47512, 47513, 47523, 47514, 47515, 47524, 47516, 47517, 47518, 47525, 47519, 47520, 47526, 47521).

7. Thalassiosira oestrupii (Ostenfeld) Hasle, Skr. U. D. Nors. Vid. Akad. Osl., p. 8, pl. 1, fig. 5-7, 1960. 
Coscinosira oestrupii Ostenfeld, Iagt. Over. Temp. Salth. Plank. Skibs., p. 52, (1899) 1900.

Fig. 22-23

Valvas circulares; superfície valvar convexa; aréolas hexagonais formando fileiras tangenciais; uma fultopórtula próxima ao centro valvar e fileira única de fultopórtulas marginais; uma rimopórtula excêntrica afastada da margem e de difícil visualização. Diâm.: 14,2-26,1 $\mu \mathrm{m} ; 5-8$ aréolas em $10 \mu \mathrm{m} ; 3$ fultopórtulas marginais em $10 \mu \mathrm{m}$.

Material examinado: Brasil. Paraná: Pontal do Paraná, rio Guaraguaçu, 16/IV/2003, P. Bigunas, T. Ludwig \& A. Campos (UPCB 47494, 47496, 47498, 47500, 47501, 47502, 47503); 24/X/2003, P. Bigunas, T. Ludwig \& J. Silva (UPCB 47509, 47510, 47511, 47512, 47513, 47514, 47515, 47524, 47516, 47517, 47518, 47525, 47519, 47520, 47526, 47521).

8. Thalassiosira proschkinae Makarova, in Makarova, Genkal \& Kuzmin, Botanicheskii Zhurnal 64: 922, pl. 1, fig. 1-7, 1979.

Fig. 24-27

Valvas circulares; superfície valvar plana; aréolas delicadas, dispostas em fileiras tangenciais; uma fultopórtula e uma rimopórtula próximas ao centro valvar; fultopórtulas marginais espaçadas. Diâm.: 5,5-8,7 $\mu \mathrm{m}$; 16-20 aréolas em $10 \mu \mathrm{m}$; 4-6 fultopórtulas marginais em $10 \mu \mathrm{m}$.

Material examinado: Brasil. Paraná: Pontal do Paraná, rio Guaraguaçu, 16/IV/2003, P. Bigunas, T. Ludwig \& A. Campos (UPCB 47496, 47498, 47499, 47500, 47501, 47502, 47503, 47506); 24/X/2003, $P$. Bigunas, T. Ludwig \& J. Silva (UPCB 47515, 47516, 47517, 47518, 47525, 47519, 47520, 47526, 47521).

9. Thalassiosira pseudonana Hasle \& Heimdal, Nova Hedwigia, 31: 565, fig. 27-28, 1970.

Fig. 28

Valvas circulares; superfície valvar plana; areolação inconspícua; fileira de fultopórtulas marginais evidente; uma rimopórtula marginal. Diâm.: 4,1 $\mu \mathrm{m}$; aréolas inconspícuas; 11 fultopórtulas marginais em $10 \mu \mathrm{m}$.

T. pseudonana é semelhante a T. guillardii Hasle, mas difere quanto à localização da rimopórtula. Em T. pseudonana a rimopórtula está localizada entre duas fultopórtulas marginais, e em $T$. guillardii na posição de uma fultopórtula marginal (Hasle \& Syvertsen 1997; Hasle 1978).

Material examinado: Brasil. Paraná: 16/IV/2003, P. Bigunas, T. Ludwig \& A. Campos (UPCB 47497); 24/X/2003, P. Bigunas, T. Ludwig \& J. Silva (UPCB 47517).
10. Thalassiosira punctigera (Castracane) Hasle, Nord. J. Bot. Phycol. 022, 3: 593-608, 1983.

Ethmodiscus punctiger Castracane, Her. Maj. Stat. Off. Lond. 2: 167, pl. 7, fig. 1, 1886.

Fig. 29-30

Valvas circulares; superfície valvar levemente convexa; aréolas hexagonais dispostas em fileiras radiais, divididas em setores; uma fultopórtula central pouco evidente e fileira única de fultopórtulas marginais rodeada por uma segunda fileira de costelas marginais delicadas; rimopórtula marginal localizada mais internamente que os demais processos valvares. Diâm.: 27,7-52,9 $\mu \mathrm{m}$; 14-20 aréolas em $10 \mu \mathrm{m}$; 3 fultopórtulas marginais em $10 \mu \mathrm{m}$.

Material examinado: Brasil. Paraná: Pontal do Paraná, rio Guaraguaçu, 16/IV/2003, P. Bigunas, T. Ludwig \& A. Campos (UPCB 47493, 47495, 47496, 47497, 47498, 47499, 47501, 47506); 24/X/2003, P. Bigunas, T. Ludwig \& J. Silva (UPCB 47509, 47510, 47511, 47512, 47513, 47523, 47514, 47515, 47524, 47516, 47517, 47518, 47525, 47519, 47526).

11. Thalassiosira tealata Takano, Bull. Tokai Reg. Fish. Res. Lab. (103): 55-63, fig. 1-17, 1980.

Fig. 31-32

Valvas circulares; superfície valvar plana; aréolas inconspícuas; uma fultopórtula próxima à aréola central; fultopórtulas marginais alongadas ultrapassando a margem valvar, extremidades das fultopórtulas lateralmente expandidas; uma rimopórtula marginal. Diâm.: 4,7 $\mu \mathrm{m}$; aréolas inconspícuas; 4 fultopórtulas em $10 \mu \mathrm{m}$.

Em 1981, Takano descreveu uma espécie muito similar a T. tealata, Thalassiosira curviseriata. Esta se distingue principalmente pela presença de uma, geralmente duas fultopórtulas próximas à aréola central e fultopórtulas marginais não tão longas quanto às de T. tealata (Hasle \& Syvertsen 1997).

Material examinado: Brasil. Paraná: Pontal do Paraná, rio Guaraguaçu, 24/X/2003, P. Bigunas, T. Ludwig \& J. Silva (UPCB 47519).

12. Thalassiosira tenera Proschkina-Lavrenko, Notul. Syst. Inst. Cryptog. URSS, 14: 33, pl. 1, fig. 1-4, pl. 2, fig. 5-7, 1961.

Fig. 33-36

Valvas circulares; superfície valvar plana; aréolas hexagonais dispostas em fileiras diagonais; aréola central delicadamente mais larga que as demais; uma fultopórtula central; fultopórtulas marginais espaçadas; rimopórtula marginal de difícil visualização. Diâm.: 7,5 ㅆm; 10-12 aréolas em $10 \mu \mathrm{m} ; 4$ fultopórtulas marginais em $10 \mu \mathrm{m}$.

Material examinado: Brasil. Paraná: Pontal do Paraná, rio Guaraguaçu, 16/IV/2003, P. Bigunas, 
T. Ludwig \& A. Campos (UPCB 47502); 24/X/2003, P. Bigunas, T. Ludwig \& J. Silva (UPCB 47526).

\section{Thalassiosira sp.}

Fig. 37-40, 63

Valvas circulares; superfície valvar sutilmente convexa; aréolas arredondadas a irregulares, maiores na área central e dispostas em fileiras radiais; fultopórtulas centrais não evidenciadas, fileira de fultopórtulas marginal interrompida por duas rimopórtulas pouco evidentes. Diâm.: 11,9-23,7 $\mu \mathrm{m}$; 12-20 aréolas em $10 \mu \mathrm{m}$; 3-6 fultopórtulas marginais em $10 \mu \mathrm{m}$.

Pela ausência de espécies com características similares - aréolas maiores na região central e duas

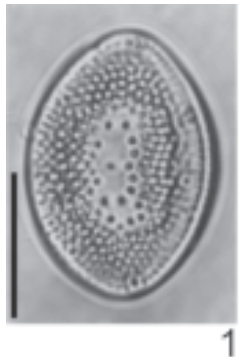

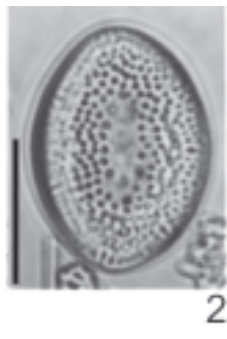

1
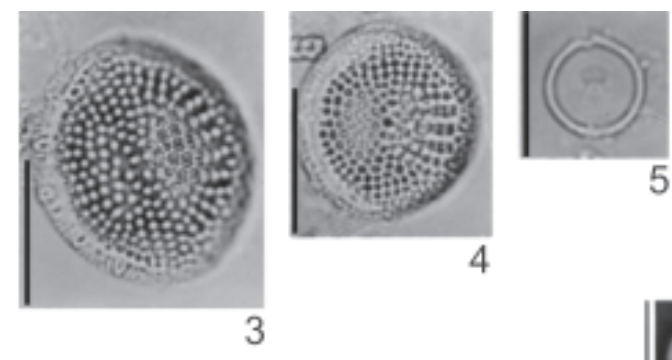

5

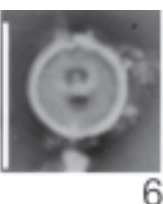

6

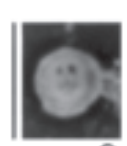

9

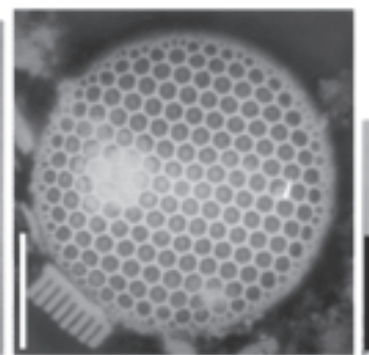

14

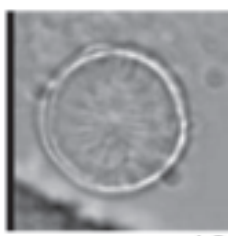

10

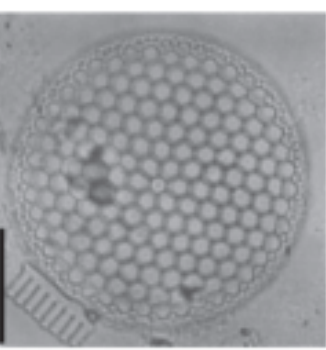

13

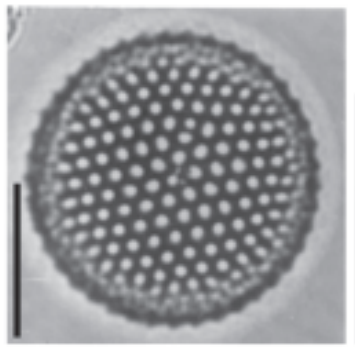

19

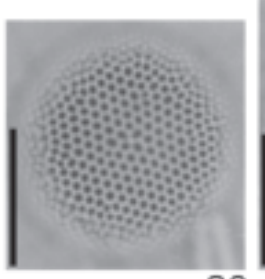

20

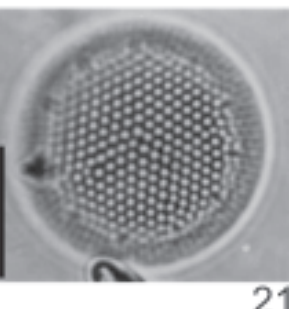

18

16

17

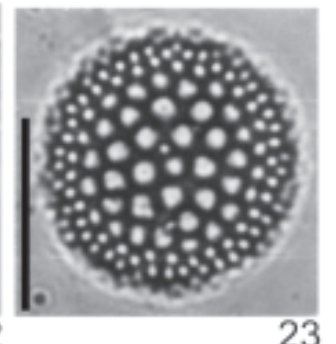

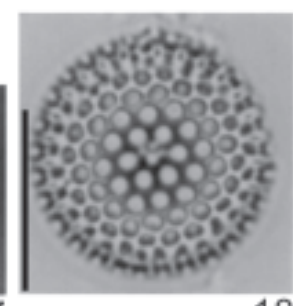

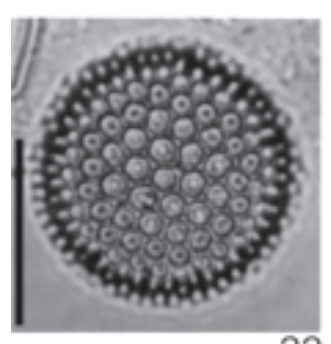

22

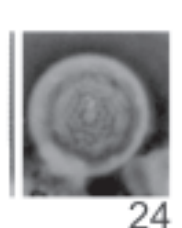

24

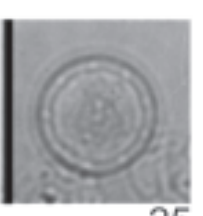

25

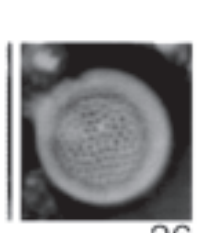

26
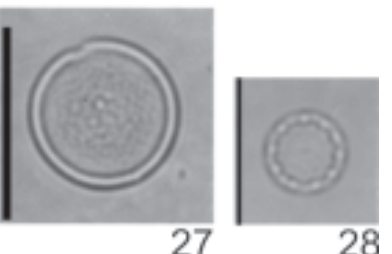

Figuras 1-28. Thalassiosirales (Diatomeae) do rio Guaraguaçu, Bacia Litorânea, PR, Brasil. 1-2. Cymatodiscus planetophorus (Meister) Hendey. 3-4. Cymatotheca weissflogii (Grunow) Hendey. 5-8. Minidiscus chilensis Rivera. 9. Minidiscus comicus Takano. 10. Thalassiocyclus lucens (Hustedt) Hakansson \& Mahood. 11. Thalassiosira decipiens (Grunow) E. Joergensen. 12. Thalassiosira eccentrica (Ehrenberg) Cleve; 13-15. Thalassiosira leptopus (Grunow) Hasle \& Fryxell. 16-17. Thalassiosira minima Gaarder. 18-19. Thalassiosira nanolineata (Mann) Fryxell \& Hasle. 20-21. Thalassiosira nordenskioeldii Cleve. 22-23. Thalassiosira oestrupii (Ostenfeld) Hasle. 24-27. Thalassiosira proschkinae Makarova. 28. Thalassiosira pseudonana Hasle \& Heimdal. Barras: $10 \mu \mathrm{m}$. 
rimopórtulas marginais - não foi possível realizar a determinação específica de Thalassiosira sp. Este táxon deve ser estudado mais detalhadamente, pois provavelmente trata-se de uma espécie ainda desconhecida para a ciência. Indivíduos observados em microscopia eletrônica de varredura apresentaram superfície valvar plana, aréolas maiores na região central da valva, grânulos delicados em toda a superfície valvar e fultopórtulas marginais com tubos externos delicados.

Material examinado: Brasil. Paraná: Pontal do Paraná, rio Guaraguaçu, 16/IV/2003, P. Bigunas, T. Ludwig \& A. Campos (UPCB 47497, 47500, 47501, 47502, 47503); 24/X/2003, P. Bigunas, T. Ludwig \& J. Silva (UPCB 47511, 47520, 47526).

\section{Tryblioptychus Hendey}

Tryblioptychus cocconeiformis (Grunow) Hendey, J.

Roy. Microscop. Soc. Ser., p. 46, pl. 2, fig. 10, 1958.

Campylodiscus cocconeiformis Grunow ex Cleve, Veg.

Exp. Vet. Iakt., p. 502, pl. 38, fig, 78, 1883.

Fig. 41

Valvas subcirculares; superfície valvar centralmente dividida em duas partes, onduladas; setores elevados delgados e depressos mais largos compostos por fileiras paralelas de pequenas aréolas; aréolas marginais maiores e alongadas, dispostas radialmente; presença de um anel marginal de fultopórtulas interrompido por uma rimopórtula de difícil visualização. Diâm.: 13,8 $\mu \mathrm{m} ; 12$ aréolas centrais em $10 \mu \mathrm{m} ; 10$ aréolas marginais em $10 \mu \mathrm{m} ; 2$ fultopórtulas em $10 \mu \mathrm{m}$.

Material examinado: Brasil. Paraná: Pontal do Paraná, rio Guaraguaçu, 16/IV/2003, P. Bigunas, T. Ludwig \& A. Campos (UPCB 47493).

\section{SKELETONEMATACEAE}

\section{Skeletonema Greville}

Skeletonema costatum (Greville) Cleve, Svensk. Vet. Akad. Handl., p. 7, 1873.

Melosira costata Greville, Trans. Microsc. Soc. Lond., p. 77, pl. 8, fig. 3-6, 1866.

Fig. 42-43

Células geralmente observadas em vista pleural formando longas cadeias retas, unidas por processos estruturais marginais; eixo pervalvar menor que o diâmetro da valva; valvas circulares; superfície valvar plana; aréolas inconspícuas; rimopórtula próxima à área central; manto com anel de fultopórtulas. Diâm.: 9,5-11,9 $\mu \mathrm{m}$; eixo pervalvar: 2,4-4 $\mu \mathrm{m}$.

Material examinado: Brasil. Paraná: Pontal do Paraná, rio Guaraguaçu, 16/IV/2003, P. Bigunas, T. Ludwig \& A. Campos (UPCB 47498, 47499, 47500, 47501, 47502, 47503, 47506); 24/X/2003, P. Bigunas, T. Ludwig \& J. Silva (UPCB 47509, 47514, 47524, 47517, 47518, 47525, 47519, 47520, 47526, 47521).

\section{STEPHANODISCACEAE}

\section{Cyclotella Kützing}

\section{Chave para as espécies de Cyclotella}

1. Uma a duas fultopórtulas na área central

2. Área central hialina ou ornamentada com linhas radiais delicadas

3. C. meneghiniana

2. Área central de aspecto rugoso

3. Fultopórtulas marginais e central evidentes; área central plana a sutilmente ondulada

1. C. atomus

3. Fultopórtulas marginais e central pouco evidentes; área central ondulada

1. Mais de sete fultopórtulas na área central

4. Câmaras marginais delicadas; duas interestrias por câmara 2. C. choctawhatcheeana

4. Câmaras marginais grosseiras; três a quatro interestrias por câmara 4. C. striata 5. C. stylorum

1. Cyclotella atomus Hustedt, Arch. Hydrobiol. p. 143, pl. 9, fig. 1-4, 1938.

Fig. 44-46

Valvas circulares; área central plana a sutilmente ondulada, de aspecto levemente rugoso; uma fultopórtula próxima ao centro da valva e uma fileira de fultopórtulas marginais conspícua; estrias marginais radiadas; rimopórtula marginal de difícil visualização, oposta a fultopórtula central. Diâm.: 4-10,3 $\mu \mathrm{m} ; 16$ estrias marginais em $10 \mu \mathrm{m}$; 4-6 fultopórtulas marginais em $10 \mu \mathrm{m}$.

Material examinado: Brasil. Paraná: Pontal do Paraná, rio Guaraguaçu, 16/IV/2003, P. Bigunas, T. Ludwig \& A. Campos (UPCB 47494, 47498, 47499, 47501, 47503, 47506); 24/X/2003, P. Bigunas, T. Ludwig \& J. Silva (UPCB 47511, 47516). 
2. Cyclotella choctawhatcheeana Prasad, in Prasad, Nienow \& Livingston, Phycol. 29(4): 419, fig. 2-26, 1990.

Fig. 47-50, 64-65

Valvas circulares; área central ondulada, de aspecto delicadamente rugoso; fultopórtula central delicada; rimopórtula e fultopórtulas marginais delicadas, pouco evidentes; estrias marginais radiadas. Diâm.: 4,7-8,7 $\mu$ m; 20-25 estrias marginais em $10 \mu \mathrm{m}$; 6-12 fultopórtulas marginais.

Håkansson et al. (1993) comentam que C. choctawhatcheeana e C. hakanssoniae Wendker são conspecíficas e que por razões de prioridade o nome C. choctawtcheeana deve ser utilizado até que estudos mais detalhados destas espécies tenham sido completados. A princípio, as duas espécies diferem ligeiramente no valor do diâmetro valvar e na localização das fultopórtulas marginais: diâmetro de 6,6-8,7 $\mu \mathrm{m}$ e uma fultopórtula a cada 2-4 costelas marginais em C. hakanssoniae, e diâmetro de 3,5-9,5 $\mu \mathrm{m}$ e uma fultopórtula a cada 3-7 costelas marginais em $C$. choctawtcheeana. Os indivíduos encontrados nas amostras do rio Guaraguaçu concordaram com a descrição de $C$. choctawhatcheeana apresentada por Prasad et al. (1990).

Material examinado: Brasil. Paraná: Pontal do Paraná, rio Guaraguaçu, 16/IV/2003, P. Bigunas, T. Ludwig \& A. Campos (UPCB 47496, 47497, 47498, 47499, 47506); 24/X/2003, P. Bigunas, T. Ludwig \& J. Silva (UPCB 47509, 47511, 47519, 47520, 47526, 47521).

3. Cyclotella meneghiniana Kützing, Bacill., p. 50, pl. 30, fig. 68, 1844.

Fig. 51-52

Valvas circulares; área central valvar plana a levemente ondulada, com linhas radiais delicadas; uma a duas fultopórtulas em uma das metades da área central; fultopórtulas e rimopórtula marginais inconspícuas; estrias marginais grosseiras, radiadas. Diâm.: 5,5-20,5 $\mu \mathrm{m}$; 7-10 estrias marginais em $10 \mu \mathrm{m}$.

Material examinado: Brasil. Paraná: Pontal do Paraná, rio Guaraguaçu, 16/IV/2003, P. Bigunas, T. Ludwig \& A. Campos (UPCB 47493, 47494, 47495, 47496, 47497, 47498, 47499, 47500, 47502, 47503, 47506); 24/X/2003, P. Bigunas, T. Ludwig \& J. Silva (UPCB 47509, 47511, 47512, 47523, 47514, 47515, 47524, 47516, 47517, 47518, 47519, 47521).

4. Cyclotella striata (Kützing) Grunow, in Cleve \& Grunow, Sven. Vet. Akad. Handl., 7(2): 119, 1880.

Coscinodiscus striatus Kützing, Bacill., p. 131, pl. 1, fig. $8,1844$.

Fig. 53-54
Valvas circulares; área central reticulada-rugosa, levemente ondulada; 2 fultopórtulas sobre uma das ondulações centrais e 7-12 fultopórtulas sobre a outra; fileira marginal de fultopórtulas inconspícua; estrias marginais radiais; alvéolos conspícuos formando um anel marginal sobre as estrias; câmaras marginais delicadas. Diâm.: 20,5-50,6 $\mu \mathrm{m}$; 8-10 estrias marginais em $10 \mu \mathrm{m}$; 3-4 câmaras em $10 \mu \mathrm{m}$; 2 interestrias por câmara.

Material examinado: Brasil. Paraná: Pontal do Paraná, rio Guaraguaçu, 16/IV/2003, P. Bigunas, T. Ludwig \& A. Campos (UPCB 47496, 47497, 47500); 24/X/2003, P. Bigunas, T. Ludwig \& J. Silva (UPCB 47509, 47510, 47511, 47512, 47513, 47523, 47514, 47515, 47524, 47516, 47518, 47525, 47519 47526).

5. Cyclotella stylorum Brigtwell, Quart. Journ. Micr. Sc. (8): 96, pr. 6, fig. 16, 1860.

Fig. 55

Valvas circulares; área central pontuada-rugosa, com ondulação pronunciada; 8-12 fultopórtulas centrais; fileira de fultopórtulas marginal inconspícua; estrias marginais radiais; câmaras marginais proeminentes. Diâm.: 33,2-56,1 $\mu \mathrm{m}$; 10-14 estrias marginais em $10 \mu \mathrm{m}$; 3-5 câmaras em 10 นm; 3-4 interestrias por câmara.

Material examinado: Brasil. Paraná: Pontal do Paraná, rio Guaraguaçu, 16/IV/2003, P. Bigunas, T. Ludwig \& A. Campos (UPCB 47495, 47497, 47498, 47499, 47500, 47501, 47502, 47503, 47506); 24/X/2003, P. Bigunas, T. Ludwig \& J. Silva (UPCB 47517, 47518, 47525, 47520, 47526).

Discostella Houk \& Klee

Chave para as espécies de Discostella

1. Fultopórtulas marginais proeminentes 1.D. pseudostelligera

1. Fultopórtulas marginais inconspícuas 2. D. stelligera

1. Discostella pseudostelligera (Hustedt) Houk \& Klee, Diatom Research 19(2): 223, 2004.

Cyclotella pseudostelligera Hustedt, Abh. Nat. Ver. Bremen, p. 581, fig. 1-2, 1

Fig. 56-57

Valvas circulares; área central plana, ornamentada por um poro central isolado rodeado por estrias radiadas em forma de roseta; anel marginal de fultopórtulas conspícuo; estrias marginais irregularmente encurtadas, radiadas. Diâm.: 4-9,5 $\mu \mathrm{m}$; 14-22 estrias marginais em $10 \mu \mathrm{m}$; 4-8 fultopórtulas marginais em $10 \mu \mathrm{m}$.

Material examinado: Brasil. Paraná: Pontal do Paraná, rio Guaraguaçu, 16/IV/2003, P. Bigunas, T. Ludwig \& A. Campos (UPCB 47493, 47495, 47496, 
47497, 47498, 47501); 24/X/2003, P. Bigunas, T. Ludwig \& J. Silva (UPCB 47509, 47510, 47511, 47512, 47513, 47523, 47514, 47515, 47524, 47516, 47517, 47518, $47519,47520,47526,47521)$.

2. Discostella stelligera (Cleve \& Grunow) Houk \& Klee, Diatom Research 19(2): 208, 2004.

Cyclotella meneghiniana var. stelligera Cleve \& Grunow in Cleve, Sven. Vet. Akad. Handl. 18(5): 22, pl. 5, fig. 63a, 1881.

Fig. 58
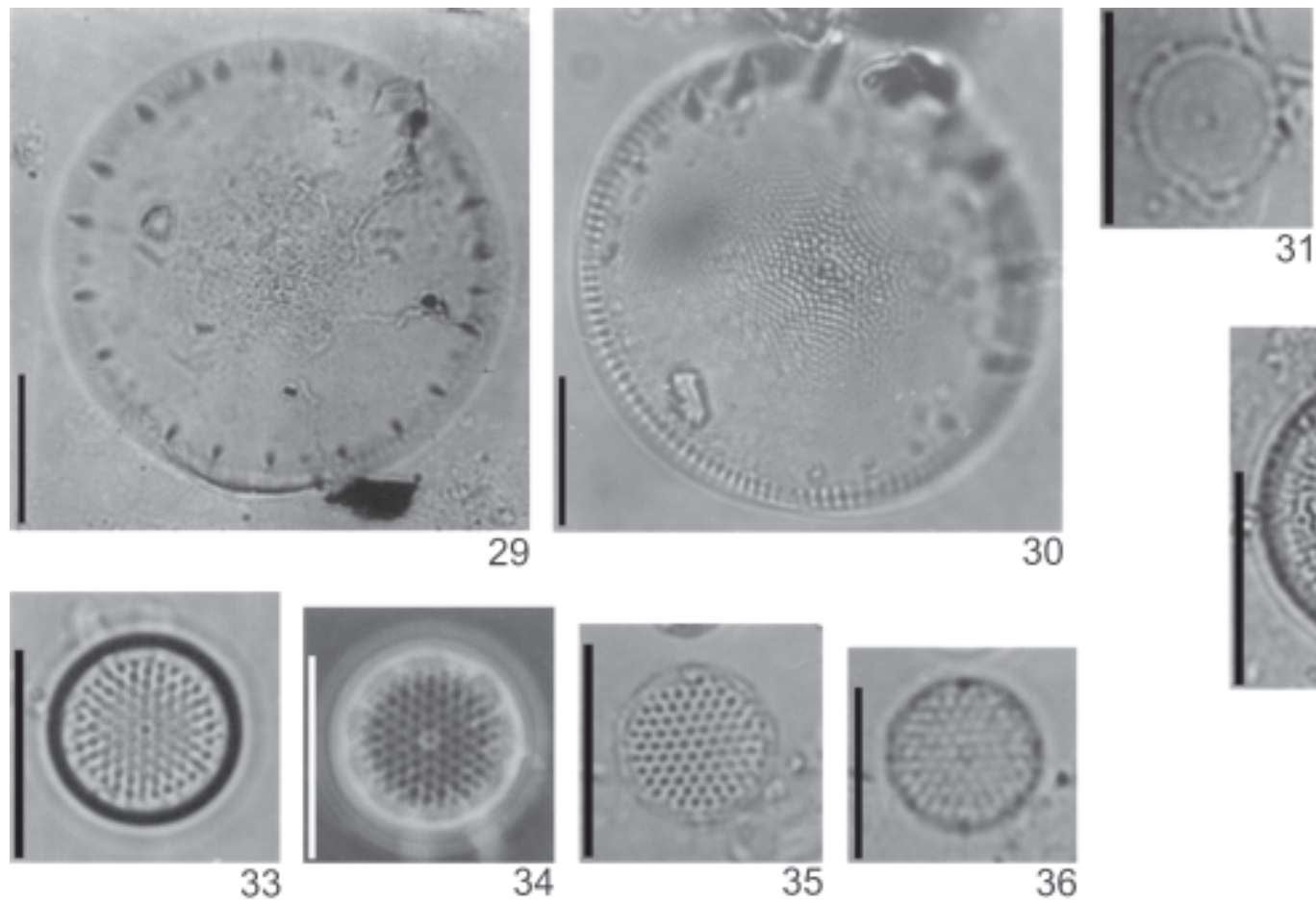

31
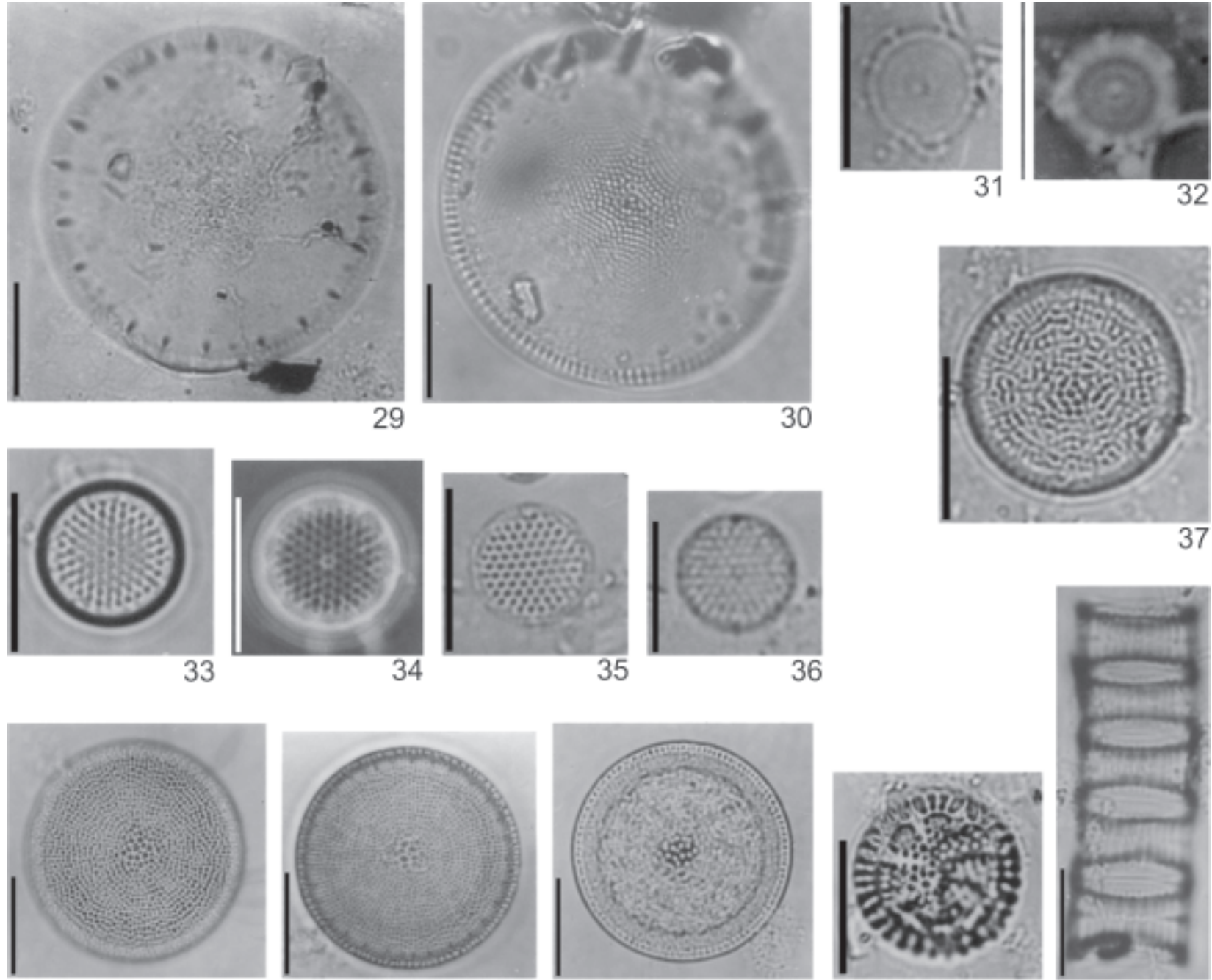

38

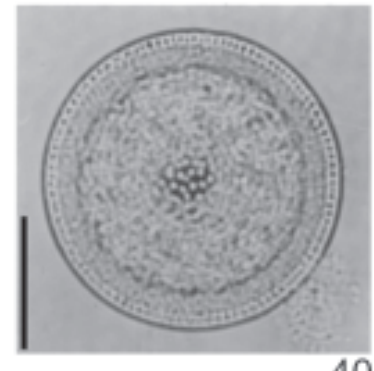

40
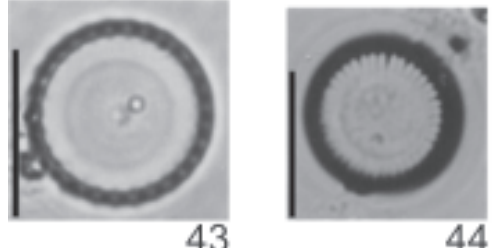

44
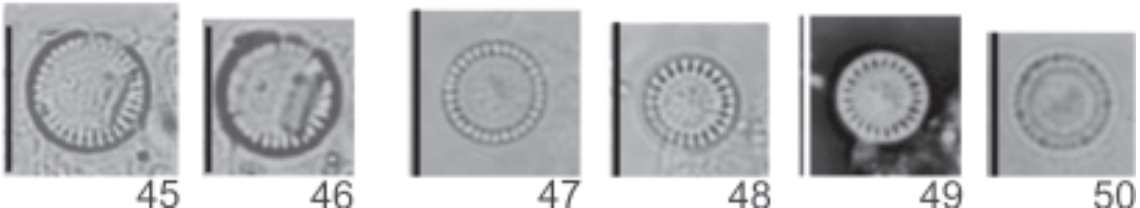

Figuras 29-50. Thalassiosirales (Diatomeae) do rio Guaraguaçu, Bacia Litorânea, PR, Brasil. 29-30. Thalassiosira punctigera (Castracane) Hasle. 31-32. Thalassiosira tealata Takano. 33-36. Thalassiosira tenera Proschkina-Lavrenko. 37-40. Thalassiosira sp. 41. Tryblioptychus cocconeiformis (Grunow) Hendey. 42-43. Skeletonema costatum (Greville) Cleve. 44-46. Cyclotella atomus Hustedt. 47-50. Cyclotella choctawhatcheeana Prasad. Barras: $10 \mu \mathrm{m}$. 
47512, 47513, 47523, 47514, 47515, 47524, 47516, $47517,47518,47525,47520,47526,47521)$.

\section{LAUDERIACEAE}

\section{Lauderia Cleve}

Lauderia annulata Cleve Bih. Svensk. Vetensk. Akad. Handl. 1(11): 8, pl. 1, fig. 7, 1973.

Fig. 59-60

Valvas circulares delicadas; superfície valvar convexa; área central diferenciada de onde partem finas costelas radiadas; presença de fultopórtulas marginais e submarginais com tubos alongados dispostas em fileiras; fultopórtulas também podem ocorrer próximo ao centro valvar; uma rimopórtula proeminente ocorre na região submarginal da valva; aréolas inconspícuas. Diâm: 26,9 $\mu \mathrm{m}$; 4 fultopórtulas marginais.

Material examinado: Brasil. Paraná: Pontal do Paraná, rio Guaraguaçu, 24/X/2003, P. Bigunas, T. Ludwig \& J. Silva (UPCB 47526).

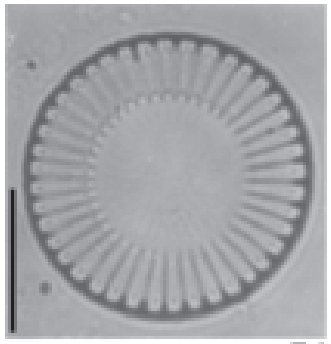

51

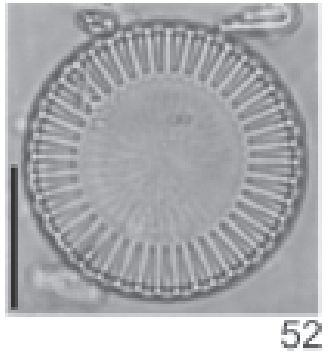

52

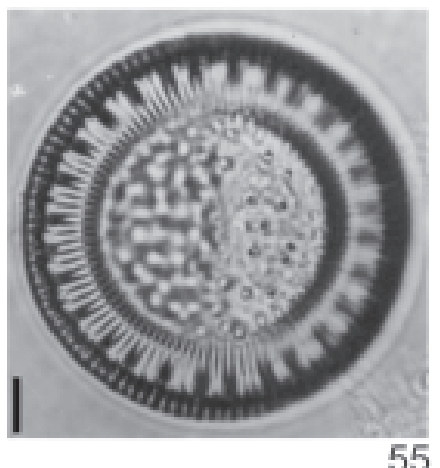

55

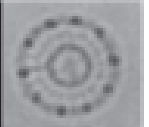

56

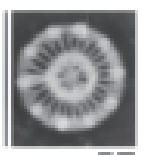

57

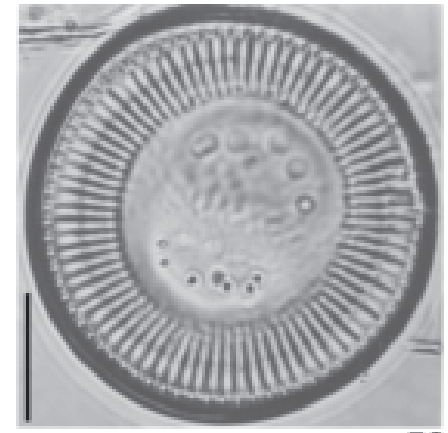

53

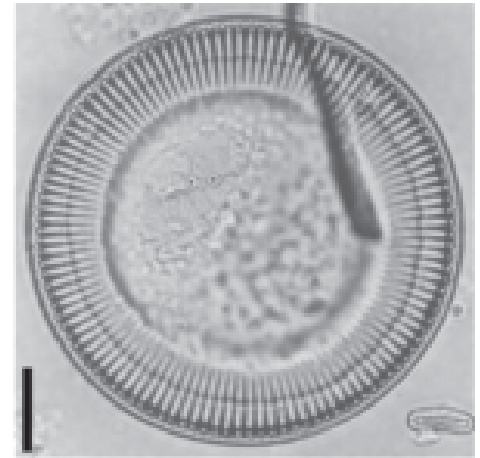

54
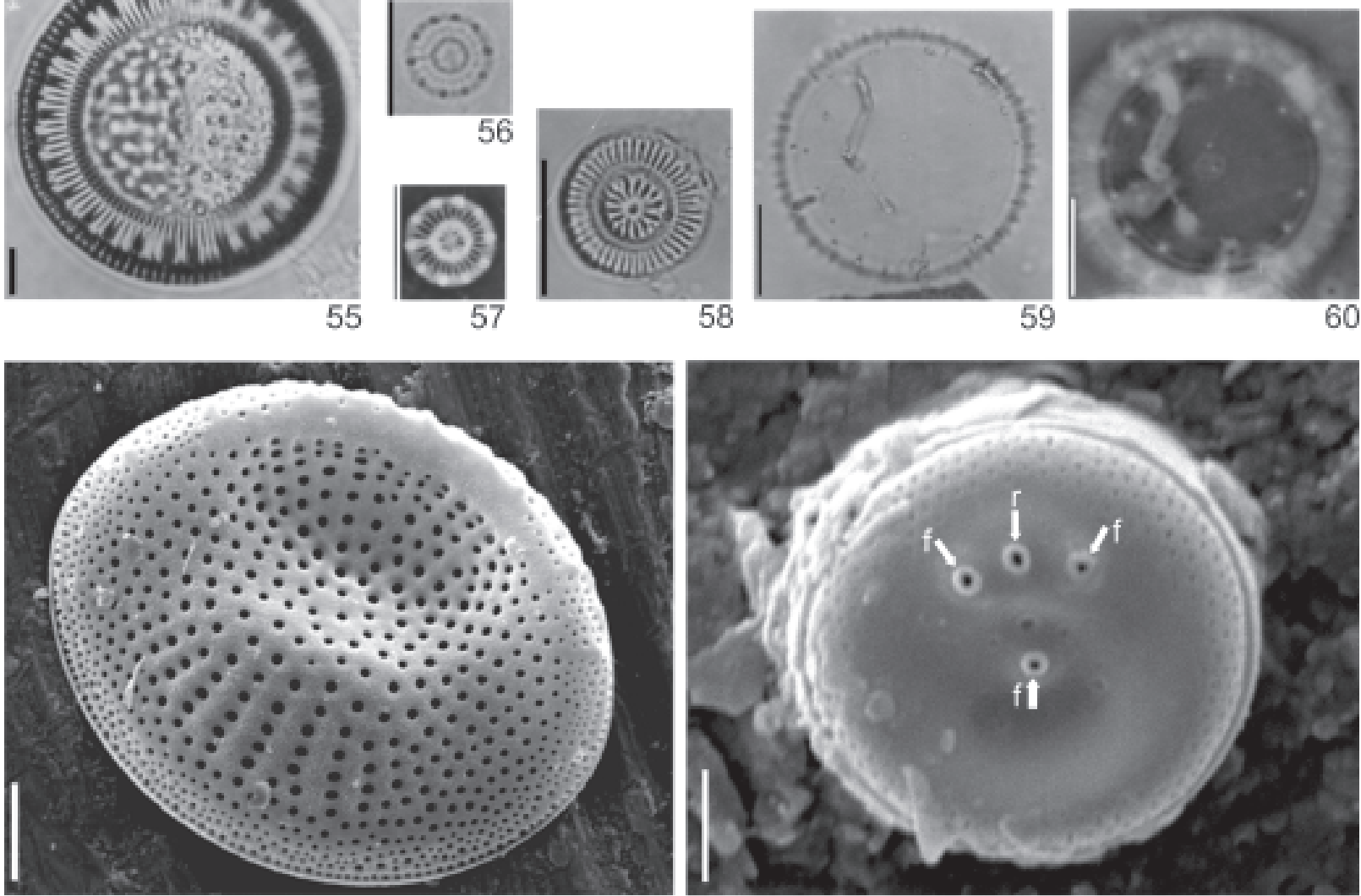

58

59

60

61

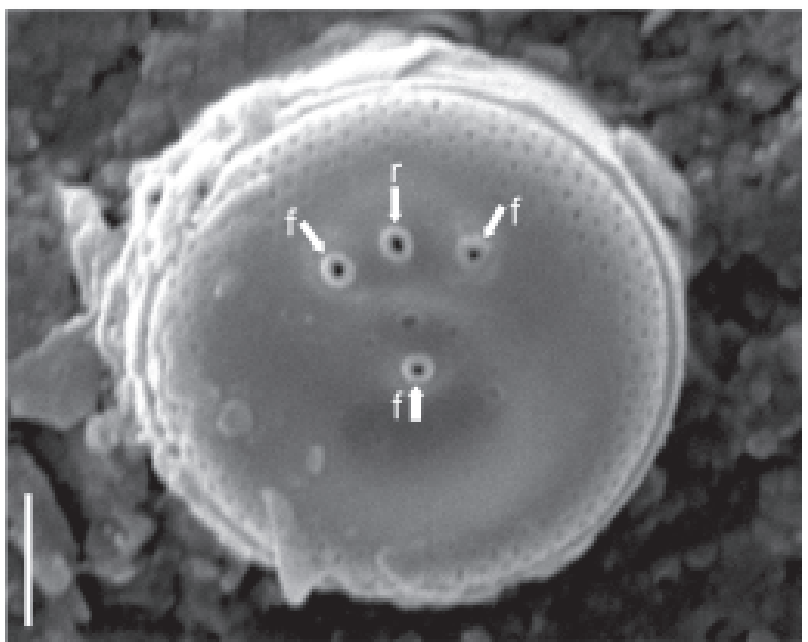

62

Figuras 51-62. Thalassiosirales (Diatomeae) do rio Guaraguaçu, Bacia Litorânea, PR, Brasil. 51-52. Cyclotella meneghiniana Kützing; 53-54. Cyclotella striata (Kützing) Grunow; 55. Cyclotella stylorum Brigtwell; 56-57. Discostella pseudostelligera (Hustedt) Houk \& Klee. 58. Discostella stelligera (Cleve \& Grunow) Houk \& Klee; 59-60. Lauderia annulata Cleve. Barras: $10 \mu \mathrm{m}$. 61. Vista geral externa da valva de Cymatotheca weissflogii (Grunow) Hendey. MEV. Escala: $5 \mu \mathrm{m}$. 62. Abertura externa da rimopórtula (seta: r) e fultopórtulas (seta: f) de Minidiscus chilensis Rivera. MEV. Escala: $1 \mu \mathrm{m}$. 

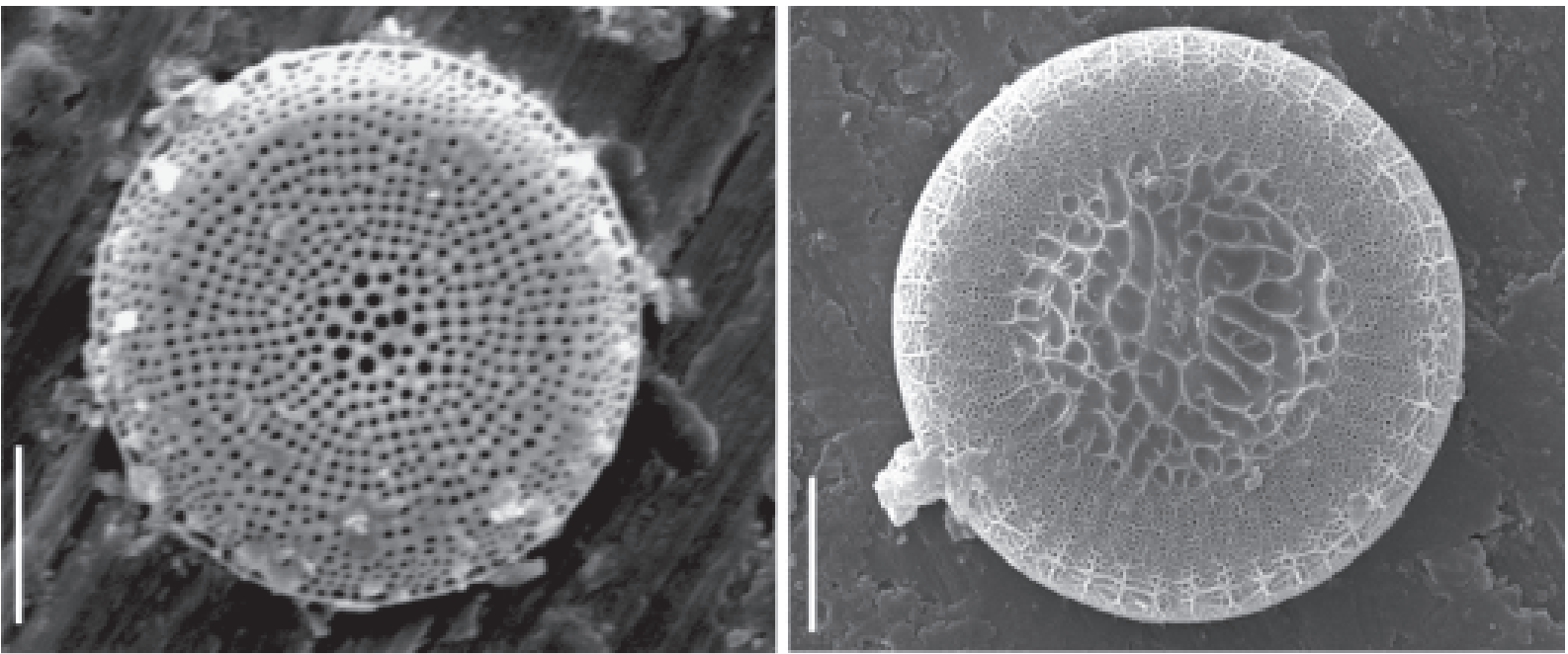

63
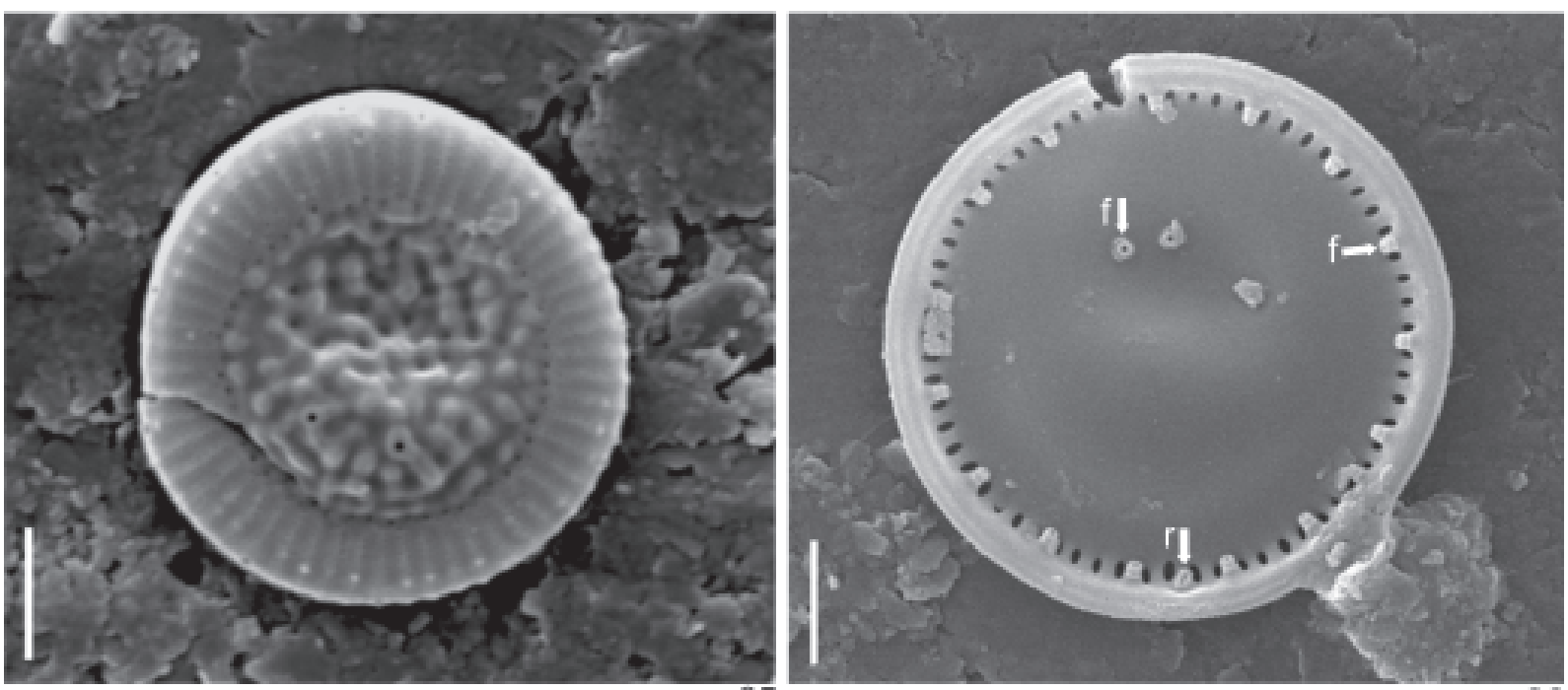

65

66

Figuras 63-66. Thalassiosirales (Diatomeae) do rio Guaraguaçu, Bacia Litorânea, PR, Brasil. 63. Vista externa da valva de Thalassiosira sp. MEV. Barra: $5 \mu \mathrm{m}$. 64. Vista externa da valva de Cyclotella striata (Kützing) Grunow. MEV. Barra: $10 \mu \mathrm{m}$. 65. Vista externa da valva de Cyclotella choctawhatcheeana Prasad. MEV. Barra: $2 \mu \mathrm{m}$. 66. Abertura interna da rimopórtula (seta: r) e das fultopórtulas (seta: f) centrais e marginais de Cyclotella choctawhatcheeana Prasad. MEV. Barra: $2 \mu \mathrm{m}$.

\section{Agradecimentos}

À Coordenação de Aperfeiçoamento de Pessoal de Nível Superior/CAPES, pela concessão de Bolsa ao primeiro Autor e aos técnicos do Centro de Microscopia Eletrônica/UFPR, pelo auxílio na obtenção das imagens do material diatomológico.

\section{Referências bibliográficas}

Barber, H.G. \& Harworth, E.Y. 1981. A Guide to the Morphology of the Diatom Frustule. Freshwater Biological Association. Ambleside, Cumbria.

Bittencourt-Oliveira, M.C. 2002. A comunidade fitoplanctônica do rio Tibagi: uma abordagem preliminar de sua diversidade. Pp. 373-402. In: M.E. Medri (ed.). A bacia do rio Tibagi, M.C. Gráfica.
Brandini, F.P.; Silva, E.T.; Pelizzari, F.M.; Fonseca, A.L.O. \& Fernandes, L.F. 2001. Production and biomass accumulation of periphytic diatoms growing on glass slides during a 1-year cycle in a subtropical estuarine environment (Bay of Paranaguá, southern Brazil). Marine Biology 138: 163-171.

Brassac, N.M. \& Ludwig, T.A.V. 2005. Amphipleuraceae e Diploneidaceae (Bacillariophyceae) da bacia do rio Iguaçu, PR, Brasil. Acta Botanica Brasilica 19: 359-368.

Brassac, N.M. \& Ludwig, T.A.V. 2006. Diatomáceas da Bacia do rio Iguaçu, Paraná, Brasil: Pinnularia e Caloneis. Hoehnea 33: 127-142.

Brassac, N.M.; Atab, D.R.; Landucci, M.; Visinoni, N.D. \& Ludwig, T.A.V. 1999. Diatomáceas cêntricas de rios na região de abrangência da Usina Hidrelétrica de Salto Caxias, PR (Bacia do Rio Iguaçu). Acta Botanica Brasilica 13: 277-289.

Cavalier-Smith, T. 1998. A revised six-kingdom system of life. Biological Reviews 73: 203-266.

Contin, L.F. 1990. Contribuição ao estudo das diatomáceas (Chrysophyta, Bacillariophyceae) na região da barragem de captação d'água do rio Iguaçu (SANEPAR), em Curitiba, Estado do Paraná, Brasil. Estudos de Biologia: 5-95. 
Fernandes, L.F.; Brandini, F.P.; Gutseit, K.S.; Fonseca, A.L.O \& Pelizzari, F.M. 1999. Bentic diatoms diatoms growing on glass slides in the Paranaguá Bay, Southern Brazil. Insula 28: $53-100$.

Håkansson, G.R. \& Mahood, A. 1993. Thalassiocyclus gen.nov.: a new genus in the Bacillariophyceae with comparison to closely related genera. Nova Hedwigia 106: 197-202.

Håkansson, H.; Hajdu, S.; Snoeijs, P. \& Loginova, L. 1993. Cyclotella hakanssoniae Wendker and its relationship to $C$. caspia Grunow and other similar brackish water Cyclotella species. Diatom Research 8: 333-347.

Hasle, G.R. 1978. Some freshwater and brackish water species of the diatom genus Thalassiosira Cleve. Phycologia 17: 263-292.

Hasle, G.R. \& Syvertsen, E.E. 1997. Marine diatoms. Pp. 5-385. In: C.R. Thomas (ed.). Identifying Marine Phytoplankton. San Diego, Academic Press.

Landucci, M. \& Ludwig, T.A.V. 2005 Diatomáceas de rios da bacia hidrográfica litorânea, PR, Brasil: Coscinodiscophyceae e Fragilariophyceae. Acta Botanica Brasilica 19: 345-357.

Leandrini, J.A.; Moreira Filho, H. \& Rodrigues, L. 2002. Espécies perifíticas de Navicula Bory de dois sistemas lóticos do município de Maringá, Estado do Paraná, Brasil. Hoehnea 29: $49-56$

Lozovei, A.L. \& Shirata, M.T. 1990. Diatomáceas (Chrysophyta, Bacillariophyceae) no rio Passaúna, Curitiba, Paraná, Brasil: levantamento qualitativo da diatomoflórula em segmento de manancial. Estudos de Biologia: 5-56.

Ludwig, T.A.V. \& Flôres, T. 1995. Diatomoflórula dos rios da região a ser inundada para a construção da Usina Hidrelétrica de Segredo, Paraná; I. Coscinodiscophyceae, Bacillariophyceae (Achnanthales e Eunotiales) e Fragilariophyceae (Meridion e Asterionella). Arquivos de Biologia e Tecnologia 38: 631-650.
Ludwig, T.A.V. \& Flôres, T. 1997. Diatomoflórula dos rios da região a ser inundada para a construção da Usina Hidrelétrica de Segredo, Paraná: Fragilariophyceae (Fragilaria e Synedra). Hoehnea 24: 55-65.

Moreira Filho, H. \& Valente-Moreira, I.M. 1981. Avaliação taxonômica e ecológica das diatomáceas (Bacillariophyceae) epífitas em algas pluricelulares obtidas nos litorais dos Estados do Paraná, Santa Catarina e São Paulo. Boletim do Museu Botânico Municipal 47:1-17.

Moreira Filho, H.; Valente-Moreira, I.M. \& Cecy, I.I.T. 1973. Diatomáceas na barragem de captação d'água (SANEPAR) do rio Iguaçu, em Curitiba, Estado do Paraná. Acta Biologica Paranaense 2: 133-145.

Prasad, A.K.S.K.; Nienow, J.A. \& Livingston, R.J. 1990. The genus Cyclotella (Bacillariophyta) in Choctawhatchee Bay, Florida, with special reference to $C$. striata and $C$. choctawhatcheeana sp. nov. Phycologia 29: 418-436.

Prasad, A.K.S.K.; Nienow, J.A. \& Livingston, R.J. 2002. The marine diatom genus Tryblioptychus Hendey (Thalassiosiraceae, Coscinodiscophyceae): fine structure, taxonomy, systematic and distribution. Diatom Research 17: 291-308.

Procopiak, L.K.; Fernandes, L.F. \& Moreira Filho, H. 2006. Diatomáceas (Bacillariophyta) marinhas e estuarinas do Paraná, Sul do Brasil: lista de espécies com ênfase em espécies nocivas. Biota Neotropica 6. Disponível em <http://www.biotaneotropica.org.br/v6n3/pt/ abstract?inventory+bn02306032006ISSN 1676-0603>

Round, F.E; Crawford, R.M. \& Mann, D.G. 1990. The diatoms. Biology and morphology of the genera. Cambridge, Cambridge University Press.

Simonsen, R. 1974. The diatom plankton of the Indian Ocean Expedition of R/V "Meteor", 1964-65 "Meteor" Forschungsergbnisse, Reihe D-Biologie: 1-66. 